\title{
PERTURBATION ANALYSIS OF THE MOORE-PENROSE INVERSE FOR A CLASS OF BOUNDED OPERATORS IN HILBERT SPACES
}

\author{
Chunyuan Deng ${ }^{\dagger}$ And Yimin Wei ${ }^{\ddagger}$
}

\begin{abstract}
Let $\mathcal{H}$ and $\mathcal{K}$ be Hilbert spaces and let $T, \widetilde{T}=T+\delta T$ be bounded operators from $\mathcal{H}$ into $\mathcal{K}$. In this article, two facts related to the perturbation bounds are studied. The first one is to find the upper bound of $\left\|\widetilde{T}^{+}-T^{+}\right\|$, which extends the results obtained by the second author and enriches the perturbation theory for the Moore-Penrose inverse. The other one is to develop explicit representations of projectors $\left\|\widetilde{T} \widetilde{T}^{+}-T T^{+}\right\|$ and $\left\|\widetilde{T}^{+} \widetilde{T}-T^{+} T\right\|$. In addition, some spectral cases related to these results are analyzed.
\end{abstract}

\section{Introduction}

Let $\mathcal{H}$ and $\mathcal{K}$ be separable complex Hilbert spaces. Denote by $\mathcal{B}(\mathcal{H}, \mathcal{K})$ the set of all bounded linear operators from $\mathcal{H}$ into $\mathcal{K}$. For an operator $A \in \mathcal{B}(\mathcal{H}, \mathcal{K})$, $\mathcal{R}(A), \mathcal{N}(A), A^{*}$ and $\|A\|$ denote the range, the null space, the adjoint and the spectral norm of $A$, respectively. The identity onto a closed subspace $\mathcal{M}$ will be denoted by $I_{\mathcal{M}}$ or $I$ if there is no confusion. For $T \in \mathcal{B}(\mathcal{H}, \mathcal{K})$, if there exists an operator $T^{+} \in \mathcal{B}(\mathcal{K}, \mathcal{H})$ satisfying the following four operator equations

$$
T T^{+} T=T, \quad T^{+} T T^{+}=T^{+}, \quad T T^{+}=\left(T T^{+}\right)^{*}, \quad T^{+} T=\left(T^{+} T\right)^{*},
$$

then $T^{+}$is called the Moore-Penrose inverse of $T$. It is well known that $T$ has the Moore-Penrose inverse if and only if $\mathcal{R}(T)$ is closed and the Moore-Penrose inverse of $T$ is unique (see $[1,2]$ ).

Let $T \in \mathcal{B}(\mathcal{H}, \mathcal{K})$ with closed range and let $\widetilde{T}=T+\delta T$ be the perturbation of $T$ by $\delta T \in \mathcal{B}(\mathcal{H}, \mathcal{K})$. The perturbation theory of a generalized inverse is

Received October 4, 2008.

2000 Mathematics Subject Classification. 47A05, 46C07, 15A09.

Key words and phrases. generalized inverse, Moore-Penrose inverse, perturbation, block operator matrix.

${ }^{\dagger}$ Partial support was provided by the National Natural Science Foundation Grants of China (No.10571113) and Shaanxi Province Education Committee (No. 09JK380).

$\ddagger$ This author is supported by the National Natural Science Foundation of China under grant 10871051, Shanghai Municipal Science \& Technology Committee under grant 09DZ2272900 and Shanghai Municipal Education Committee (Dawn Project). 
concerned with the question that if $T$ has a generalized inverse, when has $\widetilde{T}$ a generalized inverse? What are the upper bounds for $\left\|\widetilde{T}^{+}-T^{+}\right\|$?

For the perturbation in Hilbert spaces and Banach spaces, the authors in $[21,3,5,4,6,7,8,12,9,10,11]$ and $[15,19,17,16,18,20]$ have obtained some results. For example, Wei and Ding [19] gave the explicit formula for the generalized inverse of the perturbed operator under some conditions. In this paper, we will generalize the result of [19] to the cases:

Case 1: $\left(I-T T^{+}\right) \delta T T^{+} T=0$,

Case 2: $\left(I-T T^{+}\right)\left(I+\delta T T^{+}\right)^{-1} \delta T\left(I-T^{+} T\right)=0$.

Under these assumptions on perturbation operator $\delta T$, upper bounds for $\left\|\widetilde{T}^{+}\right\|$ and $\left\|\widetilde{T}^{+}-T^{+}\right\|$are presented. And the explicit representations of $\widetilde{T}^{+}$, projectors $\left\|\widetilde{T} \widetilde{T}^{+}-T T^{+}\right\|$and $\left\|\widetilde{T}^{+} \widetilde{T}-T^{+} T\right\|$ in terms of $\delta T$ and $T$ are obtained. These not only cover the special cases but also improve over the results of [19].

\section{The upper bound of $\left\|\widetilde{T}^{+}-T^{+}\right\|$}

In this section, we shall consider the problem of the upper bound of $\| \widetilde{T}^{+}-$ $T^{+} \|$, which are based on explicit expressions for $\widetilde{T}^{+}$. Let $T$ and $\delta T$ have the form

$$
T=\left(\begin{array}{cc}
0 & 0 \\
0 & T_{1}
\end{array}\right), \quad \delta T=\left(\begin{array}{ll}
\delta_{3} & \delta_{4} \\
\delta_{2} & \delta_{1}
\end{array}\right),
$$

where $T_{1}$ as an operator from $\mathcal{R}\left(T^{*}\right)$ onto $\mathcal{R}(T)$ is invertible. Throughout this paper, we need some notations. Let

$$
\begin{aligned}
\omega_{T} & =\left(I+T^{+} \delta T\right)^{-1} T^{+} \delta T\left(I-T^{+} T\right), \\
\nu_{T} & =\left(\left(I-T T^{+}\right) \delta T\right)^{+}\left(I-T T^{+}\right) \delta T \\
M & =T^{+} T\left(I+T^{+} \delta T\right)^{-1}\left(I-T^{+} T\right) \\
N & =\left(I-T T^{+}\right)\left(I+\delta T T^{+}\right)^{-1} T T^{+}
\end{aligned}
$$

We first present general expressions for $\widetilde{T}^{+}$when it is only assumed that $\delta_{4}=0$.

Theorem 1. Suppose that

$$
\left\|T^{+} \delta T\right\|<1, \quad\left(I-T T^{+}\right) \delta T T^{+} T=0 .
$$

Then $\widetilde{T}^{+}$exists if and only if $\mathcal{R}\left(\left(I-T T^{+}\right) \delta T\right)$ is closed. In this case,

$$
\begin{aligned}
\widetilde{T}^{+}= & \left(I+\left(I-\nu_{T}\right)^{*} \omega_{T}^{*}\right) T^{+} T\left[I+\omega_{T}\left(I-\nu_{T}\right)\left(I-\nu_{T}\right)^{*} \omega_{T}^{*}\right]^{-1} \\
& \times\left(I+T^{+} \delta T\right)^{-1} T^{+}\left[I-\delta T\left(\left(I-T T^{+}\right) \delta T\right)^{+}\right]+\left(\left(I-T T^{+}\right) \delta T\right)^{+}
\end{aligned}
$$


and

$$
\left\|\widetilde{T}^{+}-T^{+}\right\| \leq\left\|\left(\left(I-T T^{+}\right) \delta T\right)^{+}\right\|+\frac{\left\|T^{+} \delta T\right\|\left\|T^{+}\right\|}{1-\left\|T^{+} \delta T\right\|}\left(1+\frac{\left\|I-\delta T\left(\left(I-T T^{+}\right) \delta T\right)^{+}\right\|}{\left(1-\left\|T^{+} \delta T\right\|\right)^{2}}\right) .
$$

Proof. Let $T$ and $\delta T$ have the form as Eqn. (1), $\omega_{T}$ and $\nu_{T}$ have the form as Eqn. (2). $\left\|T^{+} \delta T\right\|<1$ implies that $I+T^{+} \delta T$ is invertible; $\left(I-T T^{+}\right) \delta T T^{+} T=$ 0 implies that $\delta_{4}=0$. Since $T^{+}=0 \oplus T_{1}^{-1}$, the invertibility of $I+T^{+} \delta T$ implies that $T_{1}+\delta_{1}=T_{1}\left(I+T_{1}^{-1} \delta_{1}\right)$ is invertible. From

$$
\left(\begin{array}{cc}
\delta_{3} & 0 \\
\delta_{2} & T_{1}+\delta_{1}
\end{array}\right)\left(\begin{array}{cc}
I & 0 \\
-\left(T_{1}+\delta_{1}\right)^{-1} \delta_{2} & \left(T_{1}+\delta_{1}\right)^{-1}
\end{array}\right)=\left(\begin{array}{cc}
\delta_{3} & 0 \\
0 & I
\end{array}\right)
$$

we get that $\mathcal{R}(\widetilde{T})$ is closed if and only if $\mathcal{R}\left(\delta_{3}\right)=\mathcal{R}\left(\left(I-T T^{+}\right) \delta T\right)$ is closed. Hence $\widetilde{T}^{+}$exists if and only if $\mathcal{R}\left(\left(I-T T^{+}\right) \delta T\right)$ is closed.

Since $\mathcal{R}\left(\delta_{3}\right)$ is closed, $\widetilde{T}$ as an operator from $\mathcal{N}\left(\delta_{3}\right) \oplus \mathcal{R}\left(\delta_{3}^{*}\right) \oplus \mathcal{R}\left(T^{*}\right)$ into $\mathcal{N}\left(\delta_{3}^{*}\right) \oplus \mathcal{R}\left(\delta_{3}\right) \oplus \mathcal{R}(T)$ has the form

$$
\widetilde{T}=\left(\begin{array}{cc}
\delta_{3} & 0 \\
\delta_{2} & T_{1}+\delta_{1}
\end{array}\right)=\left(\begin{array}{ccc}
0 & 0 & 0 \\
0 & \delta_{31} & 0 \\
\delta_{22} & \delta_{21} & T_{1}+\delta_{1}
\end{array}\right)
$$

where $\delta_{31}$ as an operator from $\mathcal{R}\left(\delta_{3}^{*}\right)$ onto $\mathcal{R}\left(\delta_{3}\right)$ is invertible. Now, we define $\Delta=\left[I+\left(T_{1}+\delta_{1}\right)^{-1} \delta_{2}\left(I-\delta_{3}^{+} \delta_{3}\right) \delta_{2}^{*}\left(T_{1}^{*}+\delta_{1}^{*}\right)^{-1}\right]^{-1}$,

$$
N=\left(\begin{array}{c}
0 \\
\delta_{22}
\end{array}\right), \quad M=\left(\begin{array}{cc}
\delta_{31} & 0 \\
\delta_{21} & T_{1}+\delta_{1}
\end{array}\right)
$$

and

$$
\begin{aligned}
\triangle^{\prime} & =\left[\left(T_{1}+\delta_{1}\right)\left(T_{1}+\delta_{1}\right)^{*}+\delta_{22} \delta_{22}^{*}\right]^{-1} \\
& =\left[\left(T_{1}+\delta_{1}\right)\left(T_{1}+\delta_{1}\right)^{*}+\delta_{2}\left(I-\delta_{3}^{+} \delta_{3}\right) \delta_{2}^{*}\right]^{-1} \\
& =\left(T_{1}^{*}+\delta_{1}^{*}\right)^{-1}\left[I+\left(T_{1}+\delta_{1}\right)^{-1} \delta_{2}\left(I-\delta_{3}^{+} \delta_{3}\right) \delta_{2}^{*}\left(T_{1}^{*}+\delta_{1}^{*}\right)^{-1}\right]^{-1}\left(T_{1}+\delta_{1}\right)^{-1} \\
& =\left(T_{1}^{*}+\delta_{1}^{*}\right)^{-1} \triangle\left(T_{1}+\delta_{1}\right)^{-1} .
\end{aligned}
$$

So

(3)

$$
\begin{aligned}
& \widetilde{T}^{+}=\left(\begin{array}{cc}
0 & 0 \\
N & M
\end{array}\right)^{*}\left[\left(\begin{array}{cc}
0 & 0 \\
N & M
\end{array}\right)\left(\begin{array}{cc}
0 & 0 \\
N & M
\end{array}\right)^{*}\right]^{+} \\
& =\left(\begin{array}{ll}
0 & N^{*}\left(N N^{*}+M M^{*}\right)^{-1} \\
0 & M^{*}\left(N N^{*}+M M^{*}\right)^{-1}
\end{array}\right) \\
& =\left(\begin{array}{ccc}
0 & -\delta_{22}^{*} \triangle^{\prime} \delta_{21} \delta_{31}^{-1} & \delta_{22}^{*} \triangle^{\prime} \\
0 & \delta_{31}^{-1} & 0 \\
0 & -\left(T_{1}+\delta_{1}\right)^{*} \triangle^{\prime} \delta_{21} \delta_{31}^{-1} & \left(T_{1}+\delta_{1}\right)^{*} \triangle^{\prime}
\end{array}\right) \\
& =\left(\begin{array}{cc}
\delta_{3}^{+}-\left(I-\delta_{3}^{+} \delta_{3}\right) \delta_{2}^{*} \triangle^{\prime} \delta_{2} \delta_{3}^{+} & \left(I-\delta_{3}^{+} \delta_{3}\right) \delta_{2}^{*} \triangle^{\prime} \\
-\left(T_{1}+\delta_{1}\right)^{*} \triangle^{\prime} \delta_{2} \delta_{3}^{+} & \left(T_{1}+\delta_{1}\right)^{*} \triangle^{\prime}
\end{array}\right)
\end{aligned}
$$




$$
\begin{aligned}
= & \left(\begin{array}{cc}
\delta_{3}^{+} & 0 \\
0 & 0
\end{array}\right)+\left(\begin{array}{cc}
I & \left(I-\delta_{3}^{+} \delta_{3}\right) \delta_{2}^{*}\left(T_{1}^{*}+\delta_{1}^{*}\right)^{-1} \\
0 & I
\end{array}\right)\left(\begin{array}{ll}
0 & 0 \\
0 & I
\end{array}\right) \\
& \times\left(\begin{array}{cc}
I & 0 \\
0 & \triangle
\end{array}\right)\left(\begin{array}{cc}
0 & 0 \\
0 & \left(T_{1}+\delta_{1}\right)^{-1}
\end{array}\right)\left(\begin{array}{cc}
0 & 0 \\
-\delta_{2} \delta_{3}^{+} & I
\end{array}\right) .
\end{aligned}
$$

Note that $\delta_{3}^{+} \oplus 0=\left(\left(I-T T^{+}\right) \delta T\right)^{+}, 0 \oplus\left(T_{1}+\delta_{1}\right)^{-1}=\left(I+T^{+} \delta T\right)^{-1} T^{+}$,

$$
\begin{aligned}
\left(\begin{array}{cc}
0 & 0 \\
0 & \left(T_{1}+\delta_{1}\right)^{-1}
\end{array}\right)\left(\begin{array}{cc}
0 & 0 \\
-\delta_{2} \delta_{3}^{+} & I
\end{array}\right)= & \left(I+T^{+} \delta T\right)^{-1} T^{+}\left[I-\delta T\left(\left(I-T T^{+}\right) \delta T\right)^{+}\right], \\
\left(\begin{array}{cc}
I & \left(I-\delta_{3}^{+} \delta_{3}\right) \delta_{2}^{*}\left(T_{1}^{*}+\delta_{1}^{*}\right)^{-1} \\
0 & I
\end{array}\right)= & {\left[I+\left(I+T^{+} \delta T\right)^{-1} T^{+} \delta T\left(I-T^{+} T\right)\right.} \\
& \left.\times\left(I-\left(\left(I-T T^{+}\right) \delta T\right)^{+}\left(I-T T^{+}\right) \delta T\right)\right]^{*} \\
= & \left(I+\omega_{T}\left(I-\nu_{T}\right)\right)^{*} \\
= & I+\left(I-\nu_{T}\right)^{*} \omega_{T}^{*}
\end{aligned}
$$

and

$$
\begin{aligned}
I \oplus \triangle= & \left\{I+\left[\left(I+T^{+} \delta T\right)^{-1} T^{+} \delta T\left(I-T^{+} T\right)\right.\right. \\
& \left.\times\left(I-\left(\left(I-T T^{+}\right) \delta T\right)^{+}\left(I-T T^{+}\right) \delta T\right)\right] \\
& \times\left[\left(I+T^{+} \delta T\right)^{-1} T^{+} \delta T\left(I-T^{+} T\right)\right. \\
& \left.\left.\times\left(I-\left(\left(I-T T^{+}\right) \delta T\right)^{+}\left(I-T T^{+}\right) \delta T\right)\right]^{*}\right\}^{-1} \\
= & \left(I+\omega_{T}\left(I-\nu_{T}\right)\left(I-\nu_{T}\right)^{*} \omega_{T}^{*}\right)^{-1} .
\end{aligned}
$$

So, we have

$$
\begin{aligned}
\widetilde{T}^{+}= & {\left[I+\omega_{T}\left(I-\nu_{T}\right)\right]^{*} T^{+} T\left[I+\omega_{T}\left(I-\nu_{T}\right)\left(I-\nu_{T}\right)^{*} \omega_{T}^{*}\right]^{-1} } \\
& \times\left(I+T^{+} \delta T\right)^{-1} T^{+}\left[I-\delta T\left(\left(I-T T^{+}\right) \delta T\right)^{+}\right]+\left(\left(I-T T^{+}\right) \delta T\right)^{+} .
\end{aligned}
$$

By using the equation that $\left(I+U V^{*}\right)^{-1}=I-U\left(I+V^{*} U\right)^{-1} V^{*}$ and $U^{*}(I+$ $\left.U U^{*}\right)^{-1}=\left(I+U^{*} U\right)^{-1} U^{*}$ we can prove that

$$
\widetilde{T}^{+}=\left[I+\omega_{T}\left(I-\nu_{T}\right)\right]^{*} T^{+} T\left[I+\omega_{T}\left(I-\nu_{T}\right)\left(I-\nu_{T}\right)^{*} \omega_{T}^{*}\right]^{-1}
$$




$$
\begin{aligned}
& \times\left(I+T^{+} \delta T\right)^{-1} T^{+}\left[I-\delta T\left(\left(I-T T^{+}\right) \delta T\right)^{+}\right]+\left(\left(I-T T^{+}\right) \delta T\right)^{+} \\
= & \left(I+T^{+} \delta T\right)^{-1}\left[I+\left(I-\nu_{T}\right)^{*} \omega_{T^{*}}^{*} \omega_{T}\left(I-\nu_{T}\right)\right]^{-1}\left(I-T^{+} T\right) \\
& {\left[\left(I+T^{+} \delta T\right)^{-1} T^{+} \delta T\left(I-\nu_{T}\right)\right]^{*}\left(I+T^{+} \delta T\right)^{-1} T^{+}\left[I-\delta T\left(\left(I-T T^{+}\right) \delta T\right)^{+}\right] } \\
& +\left(\left(I-T T^{+}\right) \delta T\right)^{+}+\left(I+T^{+} \delta T\right)^{-1} T^{+} .
\end{aligned}
$$

It follows from $\left(I+T^{+} \delta T\right)^{-1} T^{+}=T^{+}-T^{+} \delta T\left(I+T^{+} \delta T\right)^{-1} T^{+}$that

$$
\begin{aligned}
\widetilde{T}^{+}-T^{+}= & \left(\left(I-T T^{+}\right) \delta T\right)^{+}-T^{+} \delta T\left(I+T^{+} \delta T\right)^{-1} T^{+}+\left(I+T^{+} \delta T\right)^{-1} \\
& \times\left[I+\left(I-\nu_{T}\right)^{*} \omega_{T}^{*} \omega_{T}\left(I-\nu_{T}\right)\right]^{-1}\left(I-T^{+} T\right) \\
& \times\left[\left(I+T^{+} \delta T\right)^{-1} T^{+} \delta T\left(I-\nu_{T}\right)\right]^{*}\left(I+T^{+} \delta T\right)^{-1} T^{+} \\
& \times\left[I-\delta T\left(\left(I-T T^{+}\right) \delta T\right)^{+}\right] .
\end{aligned}
$$

Since $\left\|\left(I+A^{*} A\right)^{-1}\right\| \leq 1$ for arbitrary operator $A \in \mathcal{B}(\mathcal{H}, \mathcal{K})$ and $\| I-((I-$ $\left.\left.T T^{+}\right) \delta T\right)^{+}\left(I-T T^{+}\right) \delta T \| \leq 1$, we arrive at

$$
\begin{aligned}
& \left\|\widetilde{T}^{+}-T^{+}\right\| \\
\leq & \left\|\left(\left(I-T T^{+}\right) \delta T\right)^{+}\right\|+\frac{\left\|T^{+} \delta T\right\|\left\|T^{+}\right\|}{1-\left\|T^{+} \delta T\right\|}+\frac{1}{1-\left\|T^{+} \delta T\right\|} \\
& \times \frac{\left\|T^{+} \delta T\right\|}{1-\left\|T^{+} \delta T\right\|} \frac{\left\|T^{+}\right\|\left\|I-\delta T\left(\left(I-T T^{+}\right) \delta T\right)^{+}\right\|}{1-\left\|T^{+} \delta T\right\|} \\
= & \left\|\left(\left(I-T T^{+}\right) \delta T\right)^{+}\right\|+\frac{\left\|T^{+} \delta T\right\|\left\|T^{+}\right\|}{1-\left\|T^{+} \delta T\right\|}\left(1+\frac{\left\|I-\delta T\left(\left(I-T T^{+}\right) \delta T\right)^{+}\right\|}{\left(1-\left\|T^{+} \delta T\right\|\right)^{2}}\right) .
\end{aligned}
$$

In addition, if $\delta_{4}=0$ and $\delta_{2} \delta_{3}^{+}=0$, we can get the following corollary.

\section{Corollary 2. Suppose that}

$$
\left\|T^{+} \delta T\right\|<1, \quad\left(I-T T^{+}\right) \delta T T^{+} T=0, \quad T T^{+} \delta T\left[\left(I-T^{+} T\right) \delta T\right]^{+}=0 .
$$

Then $\widetilde{T}^{+}$exists if and only if $\mathcal{R}\left(\left(I-T T^{+}\right) \delta T\right)$ is closed. In this case,

$$
\widetilde{T}^{+}=\left(I+\omega_{T}^{*}\right) T^{+} T\left(I+\omega_{T} \omega_{T}^{*}\right)^{-1}\left(I+T^{+} \delta T\right)^{-1} T^{+}+\left(\left(I-T T^{+}\right) \delta T\right)^{+}
$$

with

$$
\frac{\left\|\widetilde{T}^{+}-T^{+}\right\|}{\left\|T^{+}\right\|} \leq \frac{\left\|\left(\left(I-T T^{+}\right) \delta T\right)^{+}\right\|}{\left\|T^{+}\right\|}+\frac{\left\|T^{+} \delta T\right\|}{1-\left\|T^{+} \delta T\right\|}\left[1+\frac{1}{\left(1-\left\|T^{+} \delta T\right\|\right)^{2}}\right] .
$$


Proof. Let $T$ and $\delta T$ have the form as Eqn. (1). Then

$$
\begin{gathered}
\delta T T^{+} T=T T^{+} \delta T T^{+} T \Longrightarrow \delta_{4}=0, \\
T T^{+} \delta T\left[\left(I-T^{+} T\right) \delta T\right]^{+}=0 \Longrightarrow \delta_{2} \delta_{3}^{+}=0 .
\end{gathered}
$$

So by Eqn. (3) we have

$$
\begin{aligned}
\widetilde{T}^{+}= & \left(\begin{array}{cc}
\delta_{3}^{+} & \delta_{2}^{*}\left(T_{1}^{*}+\delta_{1}^{*}\right)^{-1} \triangle_{0}\left(T_{1}+\delta_{1}\right)^{-1} \\
0 & \triangle_{0}\left(T_{1}+\delta_{1}\right)^{-1}
\end{array}\right) \\
= & \left(\begin{array}{cc}
\delta_{3}^{+} & 0 \\
0 & 0
\end{array}\right)+\left(\begin{array}{cc}
I & \delta_{2}^{*}\left(T_{1}^{*}+\delta_{1}^{*}\right)^{-1} \\
0 & I
\end{array}\right)\left(\begin{array}{cc}
0 & 0 \\
0 & I
\end{array}\right) \\
& \times\left(\begin{array}{cc}
I & 0 \\
0 & \triangle_{0}
\end{array}\right)\left(\begin{array}{cc}
0 & 0 \\
0 & \left(T_{1}+\delta_{1}\right)^{-1}
\end{array}\right),
\end{aligned}
$$

where

$$
\triangle_{0}=\left[I+\left(T_{1}+\delta_{1}\right)^{-1} \delta_{2} \delta_{2}^{*}\left(T_{1}^{*}+\delta_{1}^{*}\right)^{-1}\right]^{-1} .
$$

Note that $\delta_{3}^{+} \oplus 0=\left(\left(I-T T^{+}\right) \delta T\right)^{+}, 0 \oplus\left(T_{1}+\delta_{1}\right)^{-1}=\left(I+T^{+} \delta T\right)^{-1} T^{+}$, $\left(\begin{array}{cc}I & \delta_{2}^{*}\left(T_{1}^{*}+\delta_{1}^{*}\right)^{-1} \\ 0 & I\end{array}\right)=\left[I+\left(I+T^{+} \delta T\right)^{-1} T^{+} \delta T\left(I-T^{+} T\right)\right]^{*}=I+\omega_{T}^{*}$, and

$$
\begin{aligned}
I \oplus \triangle_{0}= & \left\{I+\left[\left(I+T^{+} \delta T\right)^{-1} T^{+} \delta T\left(I-T^{+} T\right)\right]\right. \\
& \left.\times\left[\left(I+T^{+} \delta T\right)^{-1} T^{+} \delta T\left(I-T^{+} T\right)\right]^{*}\right\}^{-1} \\
= & \left(I+\omega_{T} \omega_{T}^{*}\right)^{-1} .
\end{aligned}
$$

The result then follows from these expressions. This completes the proof.

Comparing our Theorem 1 and Corollary 2 with Theorem 2 in [19], we can see that

$$
\left(I-T T^{+}\right) \delta T=0 \Longleftrightarrow \mathcal{R}(\delta T) \subset \mathcal{R}(T)
$$

and Theorem 2 in [19] become a particular case of Theorem 1 and Corollary 2. So Theorem 1 gives an improvement over that of [19].

Corollary 3 ([19]). Suppose that

$$
\mathcal{R}(\delta T) \subset \mathcal{R}(T), \quad\left\|T^{+} \delta T\right\|<1 .
$$

Then $\widetilde{T}^{+}$exists,

$$
\widetilde{T}^{+}=\left(I+\omega_{T}^{*}\right) T^{+} T\left(I+\omega_{T} \omega_{T}^{*}\right)^{-1}\left(I+T^{+} \delta T\right)^{-1} T^{+},
$$

with

$$
\frac{\left\|\widetilde{T}^{+}-T^{+}\right\|}{\left\|T^{+}\right\|} \leq \frac{\left\|T^{+} \delta T\right\|}{1-\left\|T^{+} \delta T\right\|}\left[1+\frac{1}{\left(1-\left\|T^{+} \delta T\right\|\right)^{2}}\right] .
$$

Moreover, if $T \in \mathcal{B}(\mathcal{H}, \mathcal{K})$ is injective with closed range, then $T^{+} T=I$. The following corollary are the special case of Theorem 1, Corollary 2, and Corollary 3. 
Corollary 4 ([11]). Suppose that $T \in \mathcal{B}(\mathcal{H}, \mathcal{K})$ is injective with closed range. If $\mathcal{R}(\delta T) \subset \mathcal{R}(T)$ and $\left\|T^{+} \delta T\right\|<1$, then $\widetilde{T}^{+}$is injective with closed range. Moreover, $\mathcal{R}(\widetilde{T})=\mathcal{R}(T), \widetilde{T}^{+}=\left(I+T^{+} \delta T\right)^{-1} T^{+}=T^{+}\left(I+\delta T T^{+}\right)^{-1}$ and

$$
\frac{\left\|\widetilde{T}^{+}-T^{+}\right\|}{\left\|T^{+}\right\|} \leq \frac{\left\|T^{+} \delta T\right\|}{1-\left\|T^{+} \delta T\right\|} .
$$

Similarly, if $\delta_{2}=0$, then $\widetilde{T}^{*}$ and $T^{*}$ satisfy the conditions of Theorem 1. It is easy to see the following result holds.

Theorem 5. Suppose that

$$
\left\|\delta T T^{+}\right\|<1, \quad T T^{+} \delta T\left(I-T^{+} T\right)=0 .
$$

Then $\widetilde{T}^{+}$exists if and only if $\mathcal{R}\left(\delta T\left(I-T^{+} T\right)\right)$ is closed. In this case,

$$
\begin{aligned}
\widetilde{T}^{+}= & \left(\delta T\left(I-T^{+} T\right)\right)^{+}+\left[I-\left(\delta T\left(I-T^{+} T\right)\right)^{+} \delta T\right] T^{+}\left(I+\delta T T^{+}\right)^{-1} \\
& \times\left[I+\omega_{T}^{\prime *}\left(I-\nu_{T}^{\prime}\right)^{*}\left(I-\nu_{T}^{\prime}\right) \omega_{T}^{\prime}\right]^{-1} T T^{+}\left(I+\omega_{T}^{\prime *}\left(I-\nu_{T}^{\prime}\right)^{*}\right)
\end{aligned}
$$

and

$$
\left\|\widetilde{T}^{+}-T^{+}\right\| \leq\left\|\left(\delta T\left(I-T^{+} T\right)\right)^{+}\right\|+\frac{\left\|\delta T T^{+}\right\|\left\|T^{+}\right\|}{1-\left\|\delta T T^{+}\right\|}\left(1+\frac{\left\|I-\left(\delta T\left(I-T^{+} T\right)\right)^{+} \delta T\right\|}{\left(1-\left\|\delta T T^{+}\right\|\right)^{2}}\right),
$$

where $\omega_{T}^{\prime}=\left(I-T T^{+}\right) \delta T T^{+}\left(I+\delta T T^{+}\right)^{-1}$ and $\nu_{T}^{\prime}=\left(\delta T\left(I-T^{+} T\right)\right)(\delta T(I-$ $\left.\left.T^{+} T\right)\right)^{+}$.

\section{The bound of $\left\|\widetilde{T} \widetilde{T}^{+}-T T^{+}\right\|$}

In this section, we mainly study the perturbation on the case that $\delta_{3}=$ $\delta_{4}\left(T_{1}+\delta_{1}\right)^{-1} \delta_{2}$. The explicit representations of projectors $\left\|\widetilde{T} \widetilde{T}^{+}-T T^{+}\right\|$and $\left\|\widetilde{T}^{+} \widetilde{T}-T^{+} T\right\|$ will be establish. As we know, if $\mathcal{R}(A), \mathcal{R}(B)$ and $\mathcal{R}(A B)$ are closed, then the rule $(A B)^{+}=B^{+} A^{+}$is called the reverse order rule for the Moore-Penrose inverse (and it does not hold in general). In [1], it is shown that if $\mathcal{R}(A), \mathcal{R}(B)$ and $\mathcal{R}(A B)$ are closed, then the following statements are equivalent:

(a) $(A B)^{+}=B^{+} A^{+}$;

(b) $\mathcal{R}\left(A^{*} A B\right) \subset \mathcal{R}(B)$ and $\mathcal{R}\left(B B^{*} A^{*}\right) \subset \mathcal{R}\left(A^{*}\right)$.

Let $\omega_{T}, \nu_{T}, M$ and $N$ be defined as Eqn. (2). From above result, we can deduce the following perturbation result.

Theorem 6. Suppose that

$$
\left\|\delta T T^{+}\right\|<1, \quad\left(I-T T^{+}\right)\left(I+\delta T T^{+}\right)^{-1} \delta T\left(I-T^{+} T\right)=0 .
$$

Then $\widetilde{T}^{+}$exists. In this case,

$$
\widetilde{T}^{+}=\left(T^{+} T-M^{*}\right)\left(I+M M^{*}\right)^{-1} T^{+}\left(I+\delta T T^{+}\right)^{-1}\left(I+N^{*} N\right)^{-1}\left(T T^{+}-N^{*}\right)
$$


and

$$
\|\widetilde{T}\| \leq \frac{\left\|T^{+}\right\|}{1-\left\|\delta T T^{+}\right\|} .
$$

Proof. Let $T$ and $\delta T$ have the representations as Eqn. (1). $\left\|T^{+} \delta T\right\|<1$ implies that $T_{1}+\delta_{1}$ is invertible. If $\left(I-T T^{+}\right)\left(I+\delta T T^{+}\right)^{-1} \delta T\left(I-T^{+} T\right)=0$, then $\delta_{3}=\delta_{4}\left(T_{1}+\delta_{1}\right)^{-1} \delta_{2}$ and

$\widetilde{T}=\left(\begin{array}{cc}\delta_{4}\left(T_{1}+\delta_{1}\right)^{-1} \delta_{2} & \delta_{4} \\ \delta_{2} & T_{1}+\delta_{1}\end{array}\right)=\left(\begin{array}{cc}0 & \delta_{4} \\ 0 & T_{1}+\delta_{1}\end{array}\right)\left(\begin{array}{cc}0 & 0 \\ \left(T_{1}+\delta_{1}\right)^{-1} \delta_{2} & I\end{array}\right)$.

Note that

$$
\begin{aligned}
& M=T^{+} T\left(I+T^{+} \delta T\right)^{-1}\left(I-T^{+} T\right)=\left(\begin{array}{cc}
0 & 0 \\
-\left(T_{1}+\delta_{1}\right)^{-1} \delta_{2} & 0
\end{array}\right) \\
& N=\left(I-T T^{+}\right)\left(I+\delta T T^{+}\right)^{-1} T T^{+}=\left(\begin{array}{cc}
0 & -\delta_{4}\left(T_{1}+\delta_{1}\right)^{-1} \\
0 & 0
\end{array}\right) .
\end{aligned}
$$

Let

$$
A=\left(\begin{array}{cc}
0 & \delta_{4} \\
0 & T_{1}+\delta_{1}
\end{array}\right), \quad B=\left(\begin{array}{cc}
0 & 0 \\
\left(T_{1}+\delta_{1}\right)^{-1} \delta_{2} & I
\end{array}\right)
$$

Then

$$
\begin{aligned}
A^{+} & =\left(A^{*} A\right)^{+} A^{*} \\
& =\left(\begin{array}{cc}
0 & \delta_{4}\left(T_{1}+\delta_{1}\right)^{-1}\left[I+\left(T_{1}^{*}+\delta_{1}^{*}\right)^{-1} \delta_{4}^{*} \delta_{4}\left(T_{1}+\delta_{1}\right)^{-1}\right]^{-1}\left(T_{1}^{*}+\delta_{1}^{*}\right)^{-1} \\
0 & \quad\left[I+\left(T_{1}^{*}+\delta_{1}^{*}\right)^{-1} \delta_{4}^{*} \delta_{4}\left(T_{1}+\delta_{1}\right)^{-1}\right]^{-1}\left(T_{1}^{*}+\delta_{1}^{*}\right)^{-1}
\end{array}\right)^{*} \\
& =T^{+}\left(I+\delta T T^{+}\right)^{-1}\left(I+N^{*} N\right)^{-1}\left(T T^{+}-N^{*}\right) .
\end{aligned}
$$

and

$$
\begin{aligned}
\left\|A^{+}\right\|^{2} & =\left\|A^{+} A^{+^{*}}\right\| \\
& =\left\|\left(T_{1}+\delta_{1}\right)^{-1}\left[I+\left(T_{1}^{*}+\delta_{1}^{*}\right)^{-1} \delta_{4}^{*} \delta_{4}\left(T_{1}+\delta_{1}\right)^{-1}\right]^{-1}\left(T_{1}^{*}+\delta_{1}^{*}\right)^{-1}\right\| \\
& \leq\left\|\left(T_{1}+\delta_{1}\right)^{-1}\right\|^{2}=\left\|T^{+}\left(I+\delta T T^{+}\right)^{-1}\right\|^{2} \\
& \leq\left(\frac{\left\|T^{+}\right\|}{1-\left\|\delta T T^{+}\right\|}\right)^{2} .
\end{aligned}
$$

Similarly, we have $B^{+}=B^{*}\left(B B^{*}\right)^{+}=\left(T^{+} T-M^{*}\right)\left(I+M M^{*}\right)^{-1}$ and

$$
\left\|B^{+}\right\|^{2}=\left\|B^{+^{*}} B^{+}\right\|=\left\|\left[I+\left(T_{1}+\delta_{1}\right)^{-1} \delta_{2} \delta_{2}^{*}\left(T_{1}^{*}+\delta_{1}^{*}\right)^{-1}\right]^{-1}\right\| \leq 1 .
$$

By a direct calculation, we obtain that

$$
A^{+} A=0 \oplus I, \quad B B^{+}=0 \oplus I
$$


and

$$
A^{+} A B B^{*} A^{*}=B B^{*} A^{*}, \quad B B^{+} A^{*} A B=A^{*} A B .
$$

It implies that $\mathcal{R}\left(A^{*} A B\right) \subset \mathcal{R}(B)$ and $\mathcal{R}\left(B B^{*} A^{*}\right) \subset \mathcal{R}\left(A^{*}\right)$. Hence the reverse order rule holds,

$$
\begin{aligned}
\widetilde{T}^{+} & =(A B)^{+}=B^{+} A^{+} \\
& =\left(T^{+} T-M^{*}\right)\left(I+M M^{*}\right)^{-1} T^{+}\left(I+\delta T T^{+}\right)^{-1}\left(I+N^{*} N\right)^{-1}\left(T T^{+}-N^{*}\right)
\end{aligned}
$$

and $\|\widetilde{T}\| \leq\left\|B^{+}\right\|\left\|A^{+}\right\| \leq \frac{\left\|T^{+}\right\|}{1-\left\|\delta T T^{+}\right\|}$.

Next, we will give explicit expressions for $\left\|\widetilde{T} \widetilde{T}^{+}-T T^{+}\right\|$and $\left\|\widetilde{T}^{+} \widetilde{T}-T^{+} T\right\|$. A auxiliary result is summarized in the following lemma.

Lemma 7 ([1]). If $P$ and $Q$ are two orthogonal projections, then

$$
\|P-Q\|=\max \{\|P(I-Q)\|,\|Q(I-P)\|\} .
$$

Theorem 8. Suppose that

$$
\left\|T^{+} \delta T\right\|<1, \quad\left(I-T T^{+}\right) \delta T T^{+} T=0 .
$$

Then $\widetilde{T}^{+}$exists if and only if $\mathcal{R}\left(\left(I-T T^{+}\right) \delta T\right)$ is closed. In this case,

$$
\widetilde{T} \widetilde{T}^{+}-T T^{+}=P_{\mathcal{R}\left(\left(I-T T^{+}\right) \delta T\right)}
$$

and

$$
\begin{aligned}
& \left\|\widetilde{T}^{+} \widetilde{T}-T^{+} T\right\|^{2}
\end{aligned}
$$

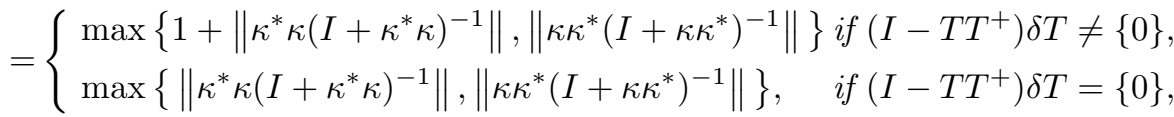

where $\kappa=\omega_{T}\left(I-\nu_{T}\right)$.

Proof. Let $T, \delta T$ and $\widetilde{T}^{+}$have the representations as Eqn. (1) and Eqn. (3). Then a direct calculation can show that $\widetilde{T} \widetilde{T}^{+}=\delta_{3} \delta_{3}^{+} \oplus I=P_{\mathcal{R}\left(\left(I-T T^{+}\right) \delta T\right)} \oplus I$ and $T T^{+}=0 \oplus I$. So the first result holds. From Eqn. (3) again we have $T^{+} T=0 \oplus I$ and $\widetilde{T}^{+} \widetilde{T}$ has the matrix representation as:

$$
\left(\begin{array}{cc}
\delta_{3}^{+} \delta_{3}+\left(I-\delta_{3}^{+} \delta_{3}\right) \delta_{2}^{*}\left(T_{1}^{*}+\delta_{1}^{*}\right)^{-1} \triangle\left(T_{1}+\delta_{1}\right)^{-1} \delta_{2}\left(I-\delta_{3}^{+} \delta_{3}\right) & \left(I-\delta_{3}^{+} \delta_{3}\right) \delta_{2}^{*}\left(T_{1}^{*}+\delta_{1}^{*}\right)^{-1} \triangle \\
\triangle\left(T_{1}+\delta_{1}\right)^{-1} \delta_{2}\left(I-\delta_{3}^{+} \delta_{3}\right) & \triangle
\end{array}\right)
$$

So we have

$$
\widetilde{T}^{+} \widetilde{T}\left(I-T^{+} T\right)=\left(\begin{array}{cc}
\delta_{3}^{+} \delta_{3}+\left(I-\delta_{3}^{+} \delta_{3}\right) \delta_{2}^{*}\left(T_{1}^{*}+\delta_{1}^{*}\right)^{-1} \triangle\left(T_{1}+\delta_{1}\right)^{-1} \delta_{2}\left(I-\delta_{3}^{+} \delta_{3}\right) & 0 \\
\triangle\left(T_{1}+\delta_{1}\right)^{-1} \delta_{2}\left(I-\delta_{3}^{+} \delta_{3}\right) & 0
\end{array}\right)
$$

and

$$
T^{+} T\left(I-\widetilde{T}^{+} \widetilde{T}\right)=\left(\begin{array}{cc}
0 & 0 \\
-\triangle\left(T_{1}+\delta_{1}\right)^{-1} \delta_{2}\left(I-\delta_{3}^{+} \delta_{3}\right) & I-\triangle
\end{array}\right),
$$

where $\triangle=\left[I+\left(T_{1}+\delta_{1}\right)^{-1} \delta_{2}\left(I-\delta_{3}^{+} \delta_{3}\right) \delta_{2}^{*}\left(T_{1}^{*}+\delta_{1}^{*}\right)^{-1}\right]^{-1}$. 
Let $X=\left(T_{1}+\delta_{1}\right)^{-1} \delta_{2}\left(I-\delta_{3}^{+} \delta_{3}\right)$ and $\kappa=\omega_{T}\left(I-\nu_{T}\right)$. By the proof of Theorem 1, we have $\|X\|=\|\kappa\|$. Hence

$$
\begin{aligned}
& \left\|\widetilde{T}^{+} \widetilde{T}\left(I-T^{+} T\right)\right\|^{2} \\
= & \left\|\left(\begin{array}{cc}
\delta_{3}^{+} \delta_{3}+\left(I-\delta_{3}^{+} \delta_{3}\right) \delta_{2}^{*}\left(T_{1}^{*}+\delta_{1}^{*}\right)^{-1} \triangle\left(T_{1}+\delta_{1}\right)^{-1} \delta_{2}\left(I-\delta_{3}^{+} \delta_{3}\right) & 0 \\
\triangle\left(T_{1}+\delta_{1}\right)^{-1} \delta_{2}\left(I-\delta_{3}^{+} \delta_{3}\right) & 0
\end{array}\right)\right\|^{2} \\
= & \left\|\left(\begin{array}{cc}
\delta_{3}^{+} \delta_{3}+X^{*}\left(I+X X^{*}\right)^{-1} X & 0 \\
\left(I+X X^{*}\right)^{-1} X & 0
\end{array}\right)\right\|^{2} \\
= & \left\|\left(\delta_{3}^{+} \delta_{3}+X^{*}\left(I+X X^{*}\right)^{-1} X\right)^{2}+X^{*}\left(I+X X^{*}\right)^{-2} X\right\| \\
= & \left\|\delta_{3}^{+} \delta_{3}+X^{*}\left(I+X X^{*}\right)^{-1} X X^{*}\left(I+X X^{*}\right)^{-1} X+X^{*}\left(I+X X^{*}\right)^{-2} X\right\| \\
= & \left\|\delta_{3}^{+} \delta_{3}+X^{*}\left(I+X X^{*}\right)^{-1} X\right\| \\
= & \left\|\delta_{3}^{+} \delta_{3}\right\|+\left\|X^{*}\left(I+X X^{*}\right)^{-1} X\right\| \\
= & \left\|\delta_{3}^{+} \delta_{3}\right\|+\left\|X^{*} X\left(I+X^{*} X\right)^{-1}\right\| \\
= & \left\{\begin{array}{cc}
1+\left\|\kappa^{*} \kappa\left(I+\kappa^{*} \kappa\right)^{-1}\right\|, & \text { if }\left(I-T T^{+}\right) \delta T \neq\{0\}, \\
\left\|\kappa^{*} \kappa\left(I+\kappa^{*} \kappa\right)^{-1}\right\|, & \text { if }\left(I-T T^{+}\right) \delta T=\{0\},
\end{array}\right.
\end{aligned}
$$

and

$$
\begin{aligned}
\left\|T^{+} T\left(I-\widetilde{T}^{+} \widetilde{T}\right)\right\|^{2} & =\left\|\left(\begin{array}{cc}
0 & 0 \\
-\triangle\left(T_{1}+\delta_{1}\right)^{-1} \delta_{2}\left(I-\delta_{3}^{+} \delta_{3}\right) & I-\triangle
\end{array}\right)\right\|^{2} \\
& =\left\|\left(\begin{array}{cc}
0 & 0 \\
\left(I+X X^{*}\right)^{-1} X & I-\left(I+X X^{*}\right)^{-1}
\end{array}\right)\right\|^{2} \\
& =\left\|\left(I+X X^{*}\right)^{-1} X X^{*}\left(I+X X^{*}\right)^{-1}+\left(I-\left(I+X X^{*}\right)^{-1}\right)^{2}\right\| \\
& =\left\|I-\left(I+X X^{*}\right)^{-1}\right\| \\
& =\left\|X X^{*}\left(I+X X^{*}\right)^{-1}\right\| \\
& =\left\|\kappa \kappa^{*}\left(I+\kappa \kappa^{*}\right)^{-1}\right\| .
\end{aligned}
$$

By Lemma 7, the result holds.

In particular, we can obtain the following corollary.

Corollary 9. Suppose that $\mathcal{R}(\delta T) \subset \mathcal{R}(T)$ and $\left\|T^{+} \delta T\right\|<1$. Then $\widetilde{T} \widetilde{T}^{+}-$ $T T^{+}=0$ and

$$
\left\|\widetilde{T}^{+} \widetilde{T}-T^{+} T\right\|^{2}=\max \left\{\left\|\omega_{T}^{*} \omega_{T}\left(I+\omega_{T}^{*} \omega_{T}\right)^{-1}\right\|,\left\|\omega_{T} \omega_{T}^{*}\left(I+\omega_{T} \omega_{T}^{*}\right)^{-1}\right\|\right\} .
$$

From Theorem 8, a continuity of the Moore-Penrose inverse can be developed.

Corollary 10. Let $T \in \mathcal{B}(\mathcal{H}, \mathcal{K})$ with the Moore-Penrose inverse $T^{+}$. Let $T_{n} \in \mathcal{B}(\mathcal{H}, \mathcal{K})$ satisfy $T_{n} \rightarrow T,\left(T_{n}-T\right) T^{+} T=T T^{+}\left(T_{n}-T\right) T^{+} T$ and $\mathcal{R}\left(\left(I-T T^{+}\right)\left(T_{n}-T\right)\right)$ closed for $n$ large enough. Then $T_{n}^{+} \rightarrow T^{+}$if and only if $\left(I-T T^{+}\right)\left(T_{n}-T\right)=0$. 
Proof. Since $T_{n} \rightarrow T$ and $T_{n}^{+} \rightarrow T^{+}$, we get $\left\|P_{\mathcal{R}\left(\left(I-T T^{+}\right)\left(T_{n}-T\right)\right)}\right\|=\| T_{n} T_{n}^{+}-$ $T T^{+} \|<1$ by Theorem 8. It implies that $P_{\mathcal{R}\left(\left(I-T T^{+}\right)\left(T_{n}-T\right)\right)}=0$. Hence $\left(I-T T^{+}\right)\left(T_{n}-T\right)=0$.

Conversely, if $\left(I-T T^{+}\right)\left(T_{n}-T\right)=0$, then Theorem 8 implies that

$$
T_{n} T_{n}^{+}-T T^{+}=0, \quad T_{n}^{+} T_{n}-T^{+} T \rightarrow 0 \text { as } n \rightarrow \infty .
$$

Since

$$
T_{n}^{+}-T^{+}=T_{n}^{+}\left(T-T_{n}\right) T^{+}+T_{n}^{+}\left(T_{n} T_{n}^{+}-T T^{+}\right)+\left(T_{n}^{+} T_{n}-T^{+} T\right) T^{+},
$$

we get $T_{n}^{+} \rightarrow T^{+}$if $T_{n} \rightarrow T$.

Theorem 11. Suppose that

$$
\left\|\delta T T^{+}\right\|<1, \quad\left(I-T T^{+}\right)\left(I+\delta T T^{+}\right)^{-1} \delta T\left(I-T^{+} T\right)=0 .
$$

Then

$$
\begin{aligned}
& \left\|\widetilde{T}^{+} \widetilde{T}-T^{+} T\right\|^{2}=\max \left\{\left\|M^{*} M\left(I+M^{*} M\right)^{-1}\right\|,\left\|\left(I+M M^{*}\right)^{-1} M M^{*}\right\|\right\}, \\
& \left\|\widetilde{T} \widetilde{T}^{+}-T T^{+}\right\|^{2}=\max \left\{\left\|N^{*} N\left(I+N^{*} N\right)^{-1}\right\|,\left\|\left(I+N N^{*}\right)^{-1} N N^{*}\right\|\right\} .
\end{aligned}
$$

Proof. Let $A$ and $B$ be defined as Eqn. (5). From Theorem 6 we get

$$
\begin{aligned}
& \widetilde{T}^{+} \widetilde{T}\left(I-T^{+} T\right)=B^{+} A^{+} A B\left(I-T^{+} T\right)=B^{+} B\left(I-T^{+} T\right) \\
= & \left(\begin{array}{cc}
\delta_{2}^{*}\left(T_{1}^{*}+\delta_{1}^{*}\right)^{-1}\left[I+\left(T_{1}+\delta_{1}\right)^{-1} \delta_{2} \delta_{2}^{*}\left(T_{1}^{*}+\delta_{1}^{*}\right)^{-1}\right]^{-1}\left(T_{1}+\delta_{1}\right)^{-1} \delta_{2} & 0 \\
{\left[I+\left(T_{1}+\delta_{1}\right)^{-1} \delta_{2} \delta_{2}^{*}\left(T_{1}^{*}+\delta_{1}^{*}\right)^{-1}\right]^{-1}\left(T_{1}+\delta_{1}\right)^{-1} \delta_{2}} & 0
\end{array}\right)
\end{aligned}
$$

and

$$
\begin{aligned}
& T^{+} T\left(I-\widetilde{T}^{+} \widetilde{T}\right)=T^{+} T\left(I-B^{+} B\right) \\
= & \left(\begin{array}{cc}
0 & -\delta_{2}^{*}\left(T_{1}^{*}+\delta_{1}^{*}\right)^{-1}\left[I+\left(T_{1}+\delta_{1}\right)^{-1} \delta_{2} \delta_{2}^{*}\left(T_{1}^{*}+\delta_{1}^{*}\right)^{-1}\right]^{-1} \\
0 & I-\left[I+\left(T_{1}+\delta_{1}\right)^{-1} \delta_{2} \delta_{2}^{*}\left(T_{1}^{*}+\delta_{1}^{*}\right)^{-1}\right]^{-1}
\end{array}\right)^{*} .
\end{aligned}
$$

Similar to the proof of Theorem 8, we get

$$
\begin{aligned}
& \left\|\widetilde{T}^{+} \widetilde{T}\left(I-T^{+} T\right)\right\|^{2} \\
= & \left\|\delta_{2}^{*}\left(T_{1}^{*}+\delta_{1}^{*}\right)^{-1}\left[I+\left(T_{1}+\delta_{1}\right)^{-1} \delta_{2} \delta_{2}^{*}\left(T_{1}^{*}+\delta_{1}^{*}\right)^{-1}\right]^{-1}\left(T_{1}+\delta_{1}\right)^{-1} \delta_{2}\right\| \\
= & \left\|M^{*}\left(I+M M^{*}\right)^{-1} M\right\|=\left\|M^{*} M\left(I+M^{*} M\right)^{-1}\right\|
\end{aligned}
$$

and

$$
\begin{aligned}
& \left\|T^{+} T\left(I-\widetilde{T}^{+} \widetilde{T}\right)\right\|^{2} \\
= & \left\|\left[I+\left(T_{1}+\delta_{1}\right)^{-1} \delta_{2} \delta_{2}^{*}\left(T_{1}^{*}+\delta_{1}^{*}\right)^{-1}\right]^{-1}\left(T_{1}+\delta_{1}\right)^{-1} \delta_{2} \delta_{2}^{*}\left(T_{1}^{*}+\delta_{1}^{*}\right)^{-1}\right\|
\end{aligned}
$$


$=\left\|\left(I+M M^{*}\right)^{-1} M M^{*}\right\|$.

Hence

$$
\left\|\widetilde{T}^{+} \widetilde{T}-T^{+} T\right\|^{2}=\max \left\{\left\|M^{*} M\left(I+M^{*} M\right)^{-1}\right\|,\left\|\left(I+M M^{*}\right)^{-1} M M^{*}\right\|\right\} .
$$

In a similar vein, we can show that

$$
\left\|\widetilde{T} \widetilde{T}^{+}-T T^{+}\right\|^{2}=\max \left\{\left\|N^{*} N\left(I+N^{*} N\right)^{-1}\right\|,\left\|\left(I+N N^{*}\right)^{-1} N N^{*}\right\|\right\} .
$$

\section{Conclusion remarks}

In this paper, we present some perturbation bounds of $\left\|\widetilde{T}^{+}-T^{+}\right\|, \| \widetilde{T} \widetilde{T}^{+}-$ $T T^{+} \|$and $\left\|\widetilde{T}^{+} \widetilde{T}-T^{+} T\right\|$ under some conditions. It is natural to ask if we can remove these restrictions, which will be the future research topic.

\section{References}

[1] R. H. Bouldin, Generalized inverses and factorizations, Recent applications of generalized inverses, pp. 233-249, Res. Notes in Math., 66, Pitman, Boston, Mass.-London, 1982.

[2] S. L. Campbell and C. D. Meyer, Generalized Inverses of Linear Transformations, Surveys and Reference Works in Mathematics, Pitman (Advanced Publishing Program), Boston, Mass.-London, 1979.

[3] G. Chen, Y. Wei, and Y. Xue, Perturbation analysis of the least squares solution in Hilbert spaces, Linear Algebra Appl. 244 (1996), 69-80.

[4] _ The generalized condition numbers of bounded linear operators in Banach spaces, J. Aust. Math. Soc. 76 (2004), no. 2, 281-290.

[5] G. Chen and Y. Xue, Perturbation analysis for the operator equation $T x=b$ in Banach spaces, J. Math. Anal. Appl. 212 (1997), no. 1, 107-125.

[6] - The expression of the generalized inverse of the perturbed operator under Type I perturbation in Hilbert spaces, Linear Algebra Appl. 285 (1998), no. 1-3, 1-6.

[7] J. Ding, New perturbation results on pseudo-inverses of linear operators in Banach spaces, Linear Algebra Appl. 362 (2003), 229-235.

[8] _ On the expression of generalized inverses of perturbed bounded linear operators in Banach spaces, Missouri J. Math. Sci. 15 (2003), no. 1, 40-47.

[9] J. Ding and L. J. Huang, On the continuity of generalized inverses of linear operators in Hilbert spaces, Linear Algebra Appl. 262 (1997), 229-242.

[10] _ Perturbation of generalized inverses of linear operators in Hilbert spaces, J. Math. Anal. Appl. 198 (1996), no. 2, 506-515.

[11] _ On the perturbation of the least squares solutions in Hilbert spaces, Linear Algebra Appl. 212/213 (1994), 487-500.

[12] J. Ding and Y. Wei, Relative errors versus residuals of approximate solutions of weighted least squares problems in Hilbert space, Comput. Math. Appl. 44 (2002), no. 3-4, 407411.

[13] C. W. Groetsch, Generalized Inverses of Linear Operators: representation and approximation, Monographs and Textbooks in Pure and Applied Mathematics, No. 37. Marcel Dekker, Inc., New York-Basel, 1977.

[14] M. Z. Nashed, Generalized Inverses and Applications, Academic Press, New York, 1976.

[15] G. W. Stewart, On the perturbation of pseudo-inverses, projections and linear least squares problems, SIAM Rev. 19 (1977), no. 4, 634-662.

[16] Y. Wei, The representation and approximation for the weighted Moore-Penrose inverse in Hilbert space, Appl. Math. Comput. 136 (2003), no. 2-3, 475-486. 
[17] Y. Wei and G. Chen, Perturbation of least squares problem in Hilbert spaces, Appl. Math. Comput. 121 (2001), no. 2-3, 177-183.

[18] Some equivalent conditions of stable perturbation of operators in Hilbert spaces, Appl. Math. Comput. 147 (2004), no. 3, 765-772.

[19] Y. Wei and J. Ding, Representations for Moore-Penrose inverses in Hilbert spaces, Appl. Math. Lett. 14 (2001), no. 5, 599-604.

[20] J. Zhou and G. Wang, Block idempotent matrices and generalized Schur complement, Appl. Math. Comput. 188 (2007), no. 1, 246-256.

[21] C. Zhu, J. Cai, and G. Chen, Perturbation analysis for the reduced minimum modulus of bounded linear operator in Banach spaces, Appl. Math. Comput. 145 (2003), no. 1, $13-21$.

Chunyuan Deng

College of Mathematics Science

South China Normal University

Guangzhou 510631, P. R. China

E-mail address: cydeng@scnu.edu.cn

YIMIN WEI

School of Mathematical Sciences

FUdAN UNIVERSITY

Shanghai, 200433, P. R. China

Key Laboratory of Mathematics for Nonlinear Sciences

(Fudan University), Ministry of Education

E-mail address: ymwei@fudan.edu.cn 